J. Austral. Math. Soc. Ser. B 29 (1987), 1-20

\title{
CHAOS IN OPTICS: \\ FIELD FLUCTUATIONS FOR A NONLINEAR OPTICAL FIBRE LOOP CLOSED BY A COUPLER
}

\author{
A. ANKIEWICZ ${ }^{1}$ AND C. PASK ${ }^{2}$
}

(Received 16 June 1986; revised 15 September 1986)

\begin{abstract}
Mathematical theories describing chaotic behaviour in physical systems are introduced by developing and reviewing applications to optical fibres. A theory is presented for laser light propagating in a loop formed by an optical fibre and an optical coupler. As the light traverses the fibre it suffers an attenuation and is subjected to a phase shift which will have a component proportional to the light intensity via the nonlinear optics Kerr effect. At each pass through the coupler, an extra fraction of laser light is coupled into the loop. The mathematical formulation leads to a two-dimensional map having a clear physical and geometrical interpretation. The complete solution is given in the linear regime and the onset of nonlinear behaviour is investigated as the laser power is increased. A variety of transitions is obtained including period doubling and iteration onto a strange attractor.
\end{abstract}

\section{Introduction}

Much of applied mathematics traditionally has dealt with deterministic systems modelled by differential and difference equations and their analytical solutions. The advent of computers and the combined numerical-analytical approach has enabled a wider range of models and solutions to be explored and has revealed new and often unexpected patterns of irregular or chaotic behaviour. Berry [5] provides an extensive and basic introduction to this subject.

\footnotetext{
'Department of Applied Mathematics, Research School of Physical Sciences, Australian National University, Canberra, A.C.T. 2600, Australia.

${ }^{2}$ Department of Mathematics, University College, Australian Defence Force Academy, Campbell, A.C.T. 2600 , Australia.

(1) Copyright Australian Mathematical Society 1987, Serial-fee code 0334-2700/87
} 
In this paper, we are concerned with some examples in fibre optics which may be used to introduce the reader to the theory of non-integrable systems, chaos and its physical ramifications. Both the continuous, or differential equation, and the discrete, or difference equation, cases can be covered.

Light propagation along an optical fibre can be described using geometric optics when the fibre parameters are such that we are in the multimode regime. In that case the probiem of finding ray paths is mathematicaily exactly equivalent to solving for the motion of a particle moving in a plane as described by classical mechanics $[1,6]$. When the fibre cross-section is distorted so that the contours of constant refractive index are no longer circular, a non-integrable problem may be generated and particular classes of rays may have extreme types of behaviour. We shall not pursue this example further here; it is set out in detail in [1], and a general discussion of optical-mechanical analogies is provided in [6].

At the other extreme - the single mode fibre-wave theory is used and the phase of a wave guided along the fibre becomes important. When such a fibre is used along with an optical coupler, a loop structure can be formed and the description of light propagation in such a system leads to a beautiful example of a discrete map or difference equation. It is our purpose to explore this example in detail, giving the complete solution in the situation where a linear problem results, and demonstrating how the onset of non-linear behaviour occurs and the effects produced. Some of the mathematics used here is already scattered throughout the literature, e.g. see papers in [7], but in this paper we provide a general development and review-by-example which may be followed by the uninitiated.

\section{The optical loop system}

The advent of low loss couplers has allowed construction of various new optical fibre devices and systems. A coupler has two input and two output ports. Light entering one port is split between the two outputs, with the splitting ratio depending on the length and optical parameters of the coupler. By joining an input and output port with a length of fibre a loop can be formed, and optical resonators, delay lines and other systems may be devised, e.g. $[8,11,15]$. The basic configuration is shown in Figure 1. In this paper we will show how the calculation of optical fields in the fibre loop leads to a mapping problem which has a clear physical interpretation, and which predicts some interesting results as the laser input power is increased.

Optical delay lines and pulse generators can be produced if a single pulse, the "signal", can be made to circulate around a loop many times. Signal attenuation due to coupler splitting and fibre loss can be compensated for by allowing the 
signal to draw energy from a counter-propagating pump beam by making use of stimulated Raman scattering. This principle has been used successfully by Desurvire et al [8]. The signal must be at a slightly longer wavelength than the pump.

In this paper we show that the signal power will follow variations in the pump power, and then study the pump power in the loop as a mapping problem. We assume that the laser is coherent enough to ensure phase integrity after light has travelled the length of the fibre loop. In this case, amplitudes of fields are added, whereas, in an incoherent case [16], intensities would be summed. (We return to this point in the conclusion.)

We show that, even if there are no fluctuations in the input pump power, the observed signal output from the system can change substantially from pulse to pulse. This can occur because of an initial transient as well as period doubling and chaotic behaviour due to the non-linear phase shift which high power light suffers when travelling along an optical fibre.

\section{Formulation}

Let us begin with the fields generated by the pump laser as shown in Figure 1. For our analysis, we take the input field $E_{1}^{(t)}$ to be constant, $E_{1}^{(t)}=E_{\text {pump }}$. We shall always assume a linearly polarized field, so that in all cases $E$ means the complex number describing the amplitude of the field, and a single pump frequency $\omega$ is involved so that a factor $\exp (i \omega t)$ is always cancelled out of the

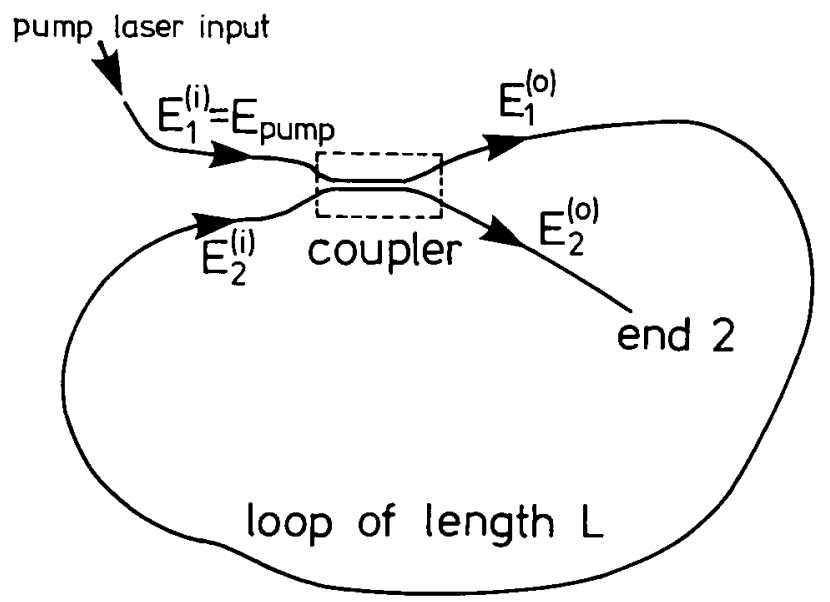

Figure 1. Experimental arrangement of fibre loop and coupler. The coupler is characterized by $C z$ where $C$ is the coupling coefficient and $z$ is the effective length. The (low intensity) signal pulse enters the system at "end 2". 
problem. Initially $E_{2}^{(i)}$ is zero and the output field $E_{1}^{(0)}$ is just the effect of the laser field passing through the coupler. Call this field $E_{1,1}^{(0)}$.

Now consider the output fields $E_{1,2}^{(0)}, E_{1,3}^{(0)}, \ldots, E_{1, n}^{(0)}, \ldots$ taken at time intervals $t$, which are just sufficient for the field to propagate around the loop and through the coupler. Thus $E_{1, n}^{(0)}$ is the effect of $E_{1}^{(i)}$, i.e. the laser field $E_{\text {pump }}$, and the input $E_{2, n-1}^{(i)}$ combining through the coupler, where $E_{2, n-1}^{(i)}$ is just $E_{1, n-1}^{(0)}$ after propagating around the loop. The objective here is the culculation of $E_{1, n}^{(0)}$.

The signal consists of a pulse injected at "end 2" in Figure 1. This is split by the coupler giving one pulse which exits at the laser input end, where it encounters a beam splitter and is detected [8], and a second pulse which enters the loop. This second pulse propagates in the opposite direction to the pump field, from which it draws power by the Raman process, and then encounters the coupler, where it suffers a splitting giving rise to a second output pulse and a new pulse entering the loop. The process repeats itself as long as sufficient power may be drawn from the pump field and thus a string of output pulses is generated at the laser input port. The technical experimental details are given in [8] and some further theory in [2], but for the purposes of this paper it suffices to note that the signal pulses can be thought of as measuring $E_{1}^{(0)}$ and so we concentrate solely on that field. Fluctuations in the desired stream of output pulses are thus related to the fluctuations in $E_{1}^{(0)}$.

\subsection{Optical loop theory}

The effect of the coupler can be represented conveniently by a $2 \times 2$ matrix operating on the incident (complex) electric fields. For a lossless coupler,

$$
\left(\begin{array}{l}
E_{1}^{(0)} \\
E_{2}^{(0)}
\end{array}\right)=e^{i \bar{\beta} z}\left(\begin{array}{cc}
\cos (C z) & i \sin (C z) \\
i \sin (C z) & \cos (C z)
\end{array}\right)\left(\begin{array}{c}
E_{1}^{(i)} \\
E_{2}^{(i)}
\end{array}\right),
$$

where $\bar{\beta}$ is the propagation constant of one fibre in isolation, $z$ is the effective coupler length, and $C$ is the coupling coefficient [2]. If there is a loss involved when light traverses the coupler, $\bar{\beta}$ will have an imaginary part $i \alpha_{c}$. The laser pump field $E_{\text {pump }}$ is substituted for $E_{1}^{(l)}$.

We can now write down the $(n+1)$ th output as defined above:

$$
E_{1, n+1}^{(0)}=e^{i \bar{\beta} z}\left\{E_{\text {pump }} \cos (C z)+i \sin (C z) E_{2, n}^{(i)}\right\} .
$$

The constant laser field $E_{\text {pump }}$ will be taken as real (this merely sets a reference zero for the phase of our fields) and so we define the real number

$$
A=E_{\text {pump }} \cos (C z) \text {. }
$$

The input field $E_{2, n}^{(i)}$ is related to $E_{1, n}^{(0)}$ as discussed above-mathematically we have

$$
E_{2, n}^{(i)}=E_{1, n}^{(0)} \exp \left\{-\alpha L+i \xi+i \phi_{N L}\right\}
$$


where $\alpha, \xi$ and $\phi_{N L}$ characterize the effect of transmission around the fibre loop. The fibre itself determines the attenuation coefficient $\alpha$, while the linear phase shift $\xi$ will be $\beta L$ for a field with propagation constant $\beta$ and loop length $L$. We may express $\beta$ as $2 \pi n_{\text {eff }} / \lambda$ where $n_{\text {eff }}$ is a refractive index derived from the basic fibre index weighted according to waveguide theory [14]. When the fields are very intense, the optical Kerr effect is important and the refractive index is modified [12]. This introduces a nonlinear term in the phase shift which we define as

$$
\phi_{N L}=q\left|E_{1, n}^{(0)}\right|^{2},
$$

where $q$ is a constant which depends on fibre properties and the nonlinear susceptibility of glass-see [12] and Appendix A.

Equation (2) may now be converted into a recurrence relation for $E_{1, n}^{(0)}$ by substituting (3), (4) and (5):

$$
E_{1, n+1}^{(0)}=e^{i \bar{\beta} z}\left\{A+i E_{1, n}^{(0)} \sin (C z) \exp \left[-\alpha L+i \xi+i q\left|E_{1, n}^{(0)}\right|^{2}\right]\right\} .
$$

\subsection{The nonlinear map}

In order to make the form of (6) clearer we replace complex numbers by two-dimensional vectors; specifically we set

$$
\begin{gathered}
\underline{A}=\left(\begin{array}{c}
A \\
0
\end{array}\right), \\
\underline{Y}_{n}=\left(\begin{array}{c}
Y_{n}^{(r)} \\
Y_{n}^{(l)}
\end{array}\right)=\left(\begin{array}{c}
\text { real part of } E_{1, n}^{(0)} \exp (-i \bar{\beta} z) \\
\text { imag. part of } E_{1, n}^{(0)} \exp (-i \bar{\beta} z)
\end{array}\right) .
\end{gathered}
$$

We also define

$$
B=e^{-\alpha L} \sin (C z)
$$

and introduce the angle $\theta_{n}$,

$$
\theta_{n}=\pi / 2+\bar{\beta} z+\xi+q\left|E_{1, n}^{(0)}\right|^{2} \equiv \xi_{L}+q\left|\underline{Y}_{n}\right|^{2},
$$

where we note that the light intensity is proportional to

$$
\left|\underline{Y}_{n}\right|^{2} \equiv \underline{Y}_{n} \cdot \underline{Y}_{n}=\left|E_{1, n}^{(0)}\right|^{2}
$$

Then (6) reduces to the form

$$
\underline{Y}_{n+1}=\underline{A}+B \underline{R}\left(\theta_{n}\right) \underline{Y}_{n},
$$

where $\underline{R}$ is the rotation matrix

$$
\underline{\underline{R}}(\theta)=\left(\begin{array}{rr}
\cos \theta & -\sin \theta \\
\sin \theta & \cos \theta
\end{array}\right) .
$$




\subsection{Features of the map}

Equation (12) is the main subject of our study; we have a map involving two-dimensional vectors, the lengths of which refer to the amplitudes of electromagnetic fields, while their directions indicate the phase. Note that from its definition (9), $|B|<1$ so that a dissipative element is involved. Since both rotations and translations are area-preserving, it is clear that the area contraction is totally controlled $b y$ the value of $B$. Evaluation of the Jacobian for the transformation confirms this, of course. We are interested mainly in the specific results generated from (12) when the physical conditions discussed earlier apply; these involve zero field in the loop when the laser is switched on; mathematically this means

$$
\underline{Y}_{0}=0, \quad \underline{Y}_{1}=\underline{A} .
$$

There are no general complete solutions of (12) except in the linear case, which we discuss in the next section. One useful general result (proved in Appendix B) concerns the invariant disc: let

$$
\rho=|A B| /(1-|B|) \text {. }
$$

Then in the $\underline{Y}$ plane there is a disc $D$ of radius $\rho$, centered on $\underline{Y}=\underline{A}$, such that if any $\underline{Y}_{n}$ is in the disc, when $\underline{Y}_{m} \in D$ for all $m \geqslant n$, i.e., once inside $D$ the iterates remain there.

Equation (12) has the nice feature that it is simply interpreted geometrically and physically as shown in Figure 2. A vector $\underline{Y}_{n}$ (representing an electric field) is rotated through $\theta_{n}$ and scaled down to a fraction $B$ of its length (representing the phase rotation and attenuation occurring during propagation along the optical fibre and through the optical coupler), and finally a constant vector $\underline{A}$ (representing a constant laser field combined with the initial field in the coupler) is added to give $\underline{Y}_{n+1}$. Figure 2(a) shows the general case and then it is clear how a fixed point $\underline{Y}_{f p}$ can occur, as in Fig. 2(b). When the nonlinear terms in $\theta_{n}$ are important we shall find a variety of behaviour including the period doubling case illustrated in Fig. 2(c).

When a fixed point solution $\underline{Y}_{f p}$ occurs, (12) with $\underline{Y}_{n}=\underline{Y}_{n+1}=\underline{Y}_{f p}$ gives

$$
\underline{Y}_{f p}=\frac{A}{1+B^{2}-2 B \cos \theta_{f p}}\left(\begin{array}{c}
1-B \cos \theta_{f p} \\
B \sin \theta_{f p}
\end{array}\right),
$$

where, from (10),

$$
\theta_{f p}=\xi_{L}+q\left|\underline{Y}_{f p}\right|^{2}
$$



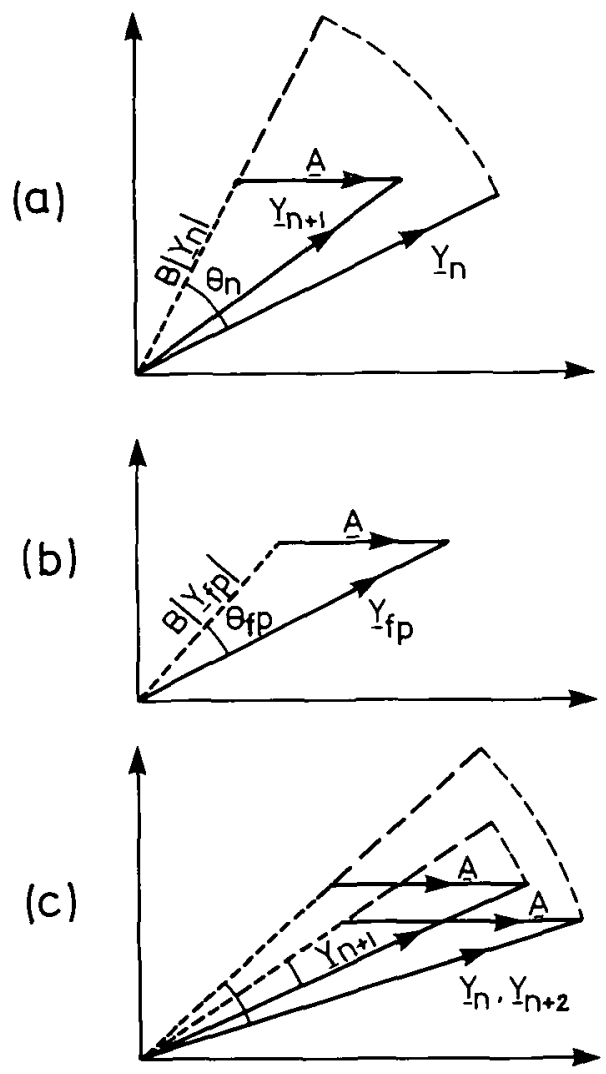

Figure 2. Geometric representation of the map given in (12).

(a) Given $\underline{Y}_{n}$, we rotate this vector by $\theta_{n}$, scale it by a factor $B$ and then add the constant vector $\underline{A}$. This produces vector $\underline{Y}_{n+1}$.

(b) Here the triangle indicates a fixed point because $\underline{Y}_{n+1}$ is the same as $\underline{Y}_{n}$.

(c) This illustrates period 2 behaviour, i.e. $\underline{Y}_{n+2}=\underline{Y}_{n} \neq \underline{Y}_{n+1}$.

Since

$$
\left|\underline{Y}_{f p}\right|^{2}=A^{2} /\left(1+B^{2}-2 B \cos \theta_{f p}\right),
$$

we may solve for $\left|\underline{Y}_{f p}\right|$. We return to the question of fixed points below.

\section{The linear case}

If we ignore nonlinear effects by setting $q=0$ in (10), we find that $\theta_{n}$ takes on the constant value $\xi_{L}$, and then our basic equation, (12), is linear. Since $|B|<1$, the contraction-mapping principle tells us that a single fixed point exists and so $\underline{Y}_{-\infty}$ is given by $\underline{Y}_{f p}$ in (15) with $\theta_{f p}=\xi_{L}$. 
In the linear case, (12) can be converted into a second order difference equation for $Y_{n}^{(r)}$, the first component of the vector $\underline{Y}_{n}$ :

$$
Y_{n+1}^{(r)}=A\left(1-B \cos \xi_{L}\right)+\left(2 B \cos \xi_{L}\right) Y_{n}^{(r)}-B^{2} Y_{n-1}^{(r)},
$$

and this can be solved by standard methods [3] to give

$$
Y_{n}^{(r)}=B^{n}\left\{C_{1} \cos \left(n \xi_{L}\right)+C_{2} \sin \left(n \xi_{L}\right)\right\}+Y_{\infty}^{(r)} .
$$

The second term is the fixed point solution and so from (15) and (16) (with $q=0$ ),

$$
Y_{\infty}^{(r)}=A\left\{1-B \cos \xi_{L}\right\} /\left\{1+B^{2}-2 B \cos \xi_{L}\right\} .
$$

The first term is the transient, and $C_{1}$ and $C_{2}$ depend on the initial conditions assumed. For our physical conditions (14),

$$
\begin{aligned}
& C_{1}=-Y_{\infty}^{(r)}, \\
& C_{2}=Y_{\infty}^{(t)} .
\end{aligned}
$$

The complete solution for $\underline{Y}_{n}$ is thus known when we note that (12) also gives for its second component,

$$
Y_{n}^{(t)}=\left\{A+B \cos \left(\xi_{L}\right) Y_{n}^{(r)}-Y_{n+1}^{(r)}\right\} / B \sin \left(\xi_{L}\right) .
$$

If we write

$$
\underline{Y}_{n}=\underline{Y}_{\infty}+\underline{w}_{n}
$$

and note that, since $\underline{Y}_{\infty}$ is the fixed point,

$$
\underline{Y}_{\infty}=\underline{A}+B \underline{R}\left(\xi_{L}\right) \underline{Y}_{\infty}
$$

then substituting into (12) gives

$$
\underline{w}_{n+1}=B \underline{R}\left(\xi_{L}\right) \underline{w}_{n} .
$$

Thus geometrically we find that the iterates spiral in towards the fixed point with constant contraction $|B|$ and rotation $\xi_{L}$ if $B>0$, and $\xi_{L}+\pi$ if $B<0$.

\subsection{Physical interpretation}

Recalling the definitions of $\underline{Y}$ and $\xi_{L}$, equations (8) and (10), allows us to translate these results back in terms of the fibre parameters and fields. We leave a detailed study to [2], but as an example we note that the steady-state power in the loop is maximised when

$$
\begin{array}{ll}
\xi_{L}=0,2 \pi, 4 \pi, \ldots & \text { if } \sin C z>0, \\
\xi_{L}=\pi, 3 \pi, 5 \pi, \ldots & \text { if } \sin C z<0,
\end{array}
$$

and in each case the power is

$$
\left|E_{1}^{(0)}\right|^{2}=\left|\underline{Y}_{\infty}\right|_{\max }^{2}=\frac{E_{\text {pump }}^{2} \cos ^{2}(C z)}{\left\{1-e^{-\alpha L}|\sin (C z)|\right\}^{2}} .
$$


The loop may be tuned to the condition (26), for example by using a polarization modulator, and if the coupler is also tuned so that

$$
|\sin (C z)|=e^{-\alpha L},
$$

then the power reaches its resonant peak value

$$
\left|\underline{Y}_{\infty}\right|_{\max }^{2}=\frac{E_{\mathrm{pump}}^{2}}{1-e^{-2 \alpha L}}
$$

This peak power relative to the pump depends only on the total fibre attenuation $\alpha L$.

\section{The nonlinear case}

An important aspect of the basic map (12) is the number of fixed point solutions which may occur and their nature. In this section we consider those points and in the next section exhibit some detailed results which are relevant to the physical system which we are modelling. The full global solution for (12) is, of course, extremely complicated.

\subsection{Fixed points}

The fixed points are given by (15) once $\theta_{f p}$ is known. Combining (16) and (17) we obtain

$$
\frac{\theta_{f p}-\xi_{L}}{q A^{2}}=\frac{1}{1+B^{2}-2 B \cos \theta_{f p}},
$$

which determines $\theta_{f p}$ in terms of the parameters $\xi_{L}, q A^{2}$ and $B$. We are particularly interested in the roles played by $A$ (which relates to the input pump laser field $E_{\text {pump }}$ as in (3)), and the loop linear phase shift $\xi_{L}$ (see (10)), which may be tuned. The two terms in (30) are shown in Figure 3 for fixed $B$ : their intersections determine $\theta_{f p}$. We observe that the number of fixed points can be varied by changing $A$ and $\xi_{L}$.

In general there is an odd number of fixed point solutions, $\underline{Y}_{f p}$, of equation (12) although in special cases two points may coalesce (see Figure 3(a)). For low values of the pump field $E_{\text {pump }}$ there is only one solution. As $A$ (i.e. $E_{\text {pump }}$ ) is increased, the number of solutions increases to $3,5,7, \ldots$ and so on, although not necessarily in a monotonic fashion. However, not all of these are stable. At a given $E_{\text {pump }}$ there may be only one or two stable fixed points, or indeed, none at all. 

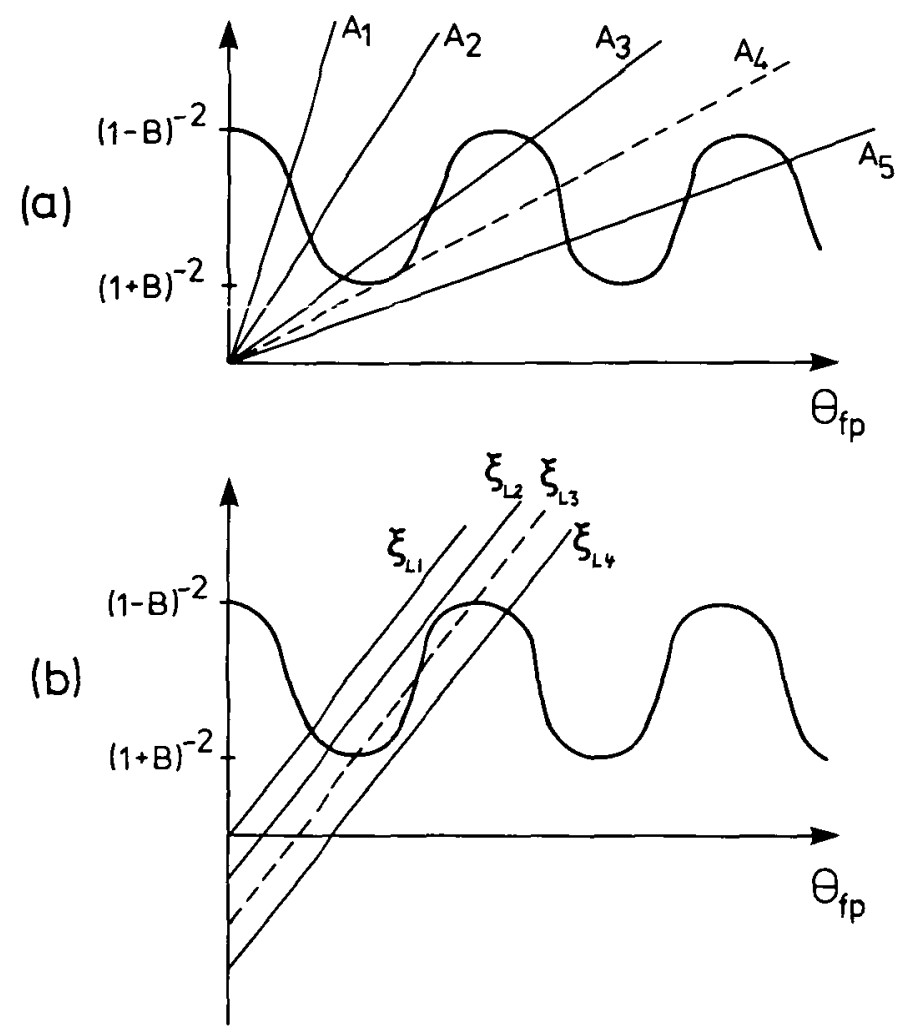

Figure 3. Graphical solution of (30) to find $\theta_{f p}$ (schematic). With $q$ and $B$ fixed, the left and right sides of the equation are plotted; intersections then lead to the fixed points.

(a) $\xi_{L}$ is set to zero; the straight lines are for various values of $A$ such that $A_{i+1}>A_{i}$; the dashed curve is the special case.

(b) Here $A$ is fixed and increasing values of $\xi_{L}$ label the straight lines, i.e. $\left(\xi_{L}\right)_{t+1}>\left(\xi_{L}\right)_{t}$.

\subsection{Stability}

Suppose $\underline{Y}_{n} \rightarrow \underline{Y}_{\infty}=\underline{Y}_{f p}$ for large $n$, i.e., that a fixed point exists. We can now linearize the map in the vicinity of this fixed point. We define

$$
\underline{w}_{n}=\underline{Y}_{n}-\underline{Y}_{\infty}
$$

and consider the case when $\left|\underline{w}_{n}\right|$ is very small. Linearizing (12) we obtain

$$
\underline{w}_{n+1}=B \underline{\underline{R}}\left(\theta_{\infty}\right) \underline{\underline{M}}_{\underline{n}},
$$

where $\theta_{\infty}$ is given by (10) and

$$
\underline{\underline{M}}=\left(\begin{array}{cc}
1-p & -2 q\left(Y_{\infty}^{(i)}\right)^{2} \\
2 q\left(Y_{\infty}^{(r)}\right)^{2} & 1+p
\end{array}\right)
$$


with

$$
p=2 q Y_{\infty}^{(r)} Y_{\infty}^{(i)} \text { and } \underline{Y}_{\infty}=\left(\begin{array}{c}
Y_{\infty}^{(r)} \\
Y_{\infty}^{(i)}
\end{array}\right)
$$

Hence

$$
\underline{w}_{n+1}=\left(\begin{array}{cc}
J_{1} & J_{2} \\
J_{3} & J_{4}
\end{array}\right) \underline{w}_{n}=\underline{J}_{\underline{w}},
$$

where the matrix entries are

$$
\begin{aligned}
& J_{1}=B\left[(1-p) \cos \theta_{\infty}-2 q\left(Y_{\infty}^{(r)}\right)^{2} \sin \theta_{\infty}\right], \\
& J_{2}=-B\left[(1+p) \sin \theta_{\infty}+2 q\left(Y_{\infty}^{(i)}\right)^{2} \cos \theta_{\infty}\right], \\
& J_{3}=B\left[(1-p) \sin \theta_{\infty}+2 q\left(Y_{\infty}^{(r)}\right)^{2} \cos \theta_{\infty}\right], \\
& J_{4}=B\left[(1+p) \cos \theta_{\infty}-2 q\left(Y_{\infty}^{(i)}\right)^{2} \sin \theta_{\infty}\right] .
\end{aligned}
$$

A fixed point is stable if both eigenvalues of $\underline{\underline{J}}$ are less than one in absolute value [4]. The eigenvalues are

$$
\lambda=J_{p} \pm\left(J_{p}^{2}-B^{2}\right)^{1 / 2},
$$

where

$$
J_{p}=\frac{1}{2}\left(J_{1}+J_{4}\right)
$$

If $J_{p}^{2}<B^{2}$ then the eigenvalues are complex, and $|\lambda|^{2}=B^{2}$; this is less than one, so the fixed point is stable.

If $J_{p}^{2}>B^{2}$ the eigenvalues are real, and $\underline{w}$ approaches zero along the eigenvector corresponding to the eigenvalue of larger absolute value. When one eigenvalue reaches 1 or -1 the fixed point becomes unstable and we get period 2 behaviour. The constraint that the eigenvalues are less than one in absolute value leads to the condition

$$
2\left|J_{p}\right|<1+B^{2}
$$

or

$$
\left.2\left|\cos \theta_{\infty}-q\right| \underline{Y}_{\infty}\right|^{2} \sin \theta_{\infty}\left|<\left(1+B^{2}\right) /\right| B \mid
$$

\subsection{Types of behaviour}

The above results show how the number of fixed points and their stability may be calculated as the system parameters $q, A, B$ and $\xi_{L}$ vary. For small values of $A$ we find one fixed point, but in general there may be more. A fixed point may become unstable or it may disappear as parameters are changed; Figure 3(a) shows that increasing $A$ from $A_{3}$ to $A_{4}$ causes two fixed points to coalesce, and then increasing $A$ beyond $A_{4}$ removes this point. 
We are particularly interested in results generated by $\underline{Y}_{0}=0$ and the fixed points reached therefrom. We then have the possibility that the fixed point reached may vary as the system parameters are changed and so a jump in behaviour type can occur.

The above changes are usually followed by results which are specific to nonlinear map iterations. These include the appearance of period two type behaviour and strange attractors, as we shall illustrate in the next section.

\section{Results}

Results are generated numerically using the initial conditions (14) in the map (12). As a first example, we show in Figure 4 values of the intensity $\left|Y_{n}\right|^{2}$ for fixed loop and coupler parameters for three values of the pump field. Figure 4(a) shows the type of behaviour expected in the linear regime or low pump power case; there is a transient and then the constant or fixed point solution. If the pump power is increased, the transient is now followed by period 2 behaviour as shown in Figure $4(\mathrm{~b})$. For even larger values of $E_{\text {pump }}$, a chaotic output is obtained, as illustrated in Figure 4(c).

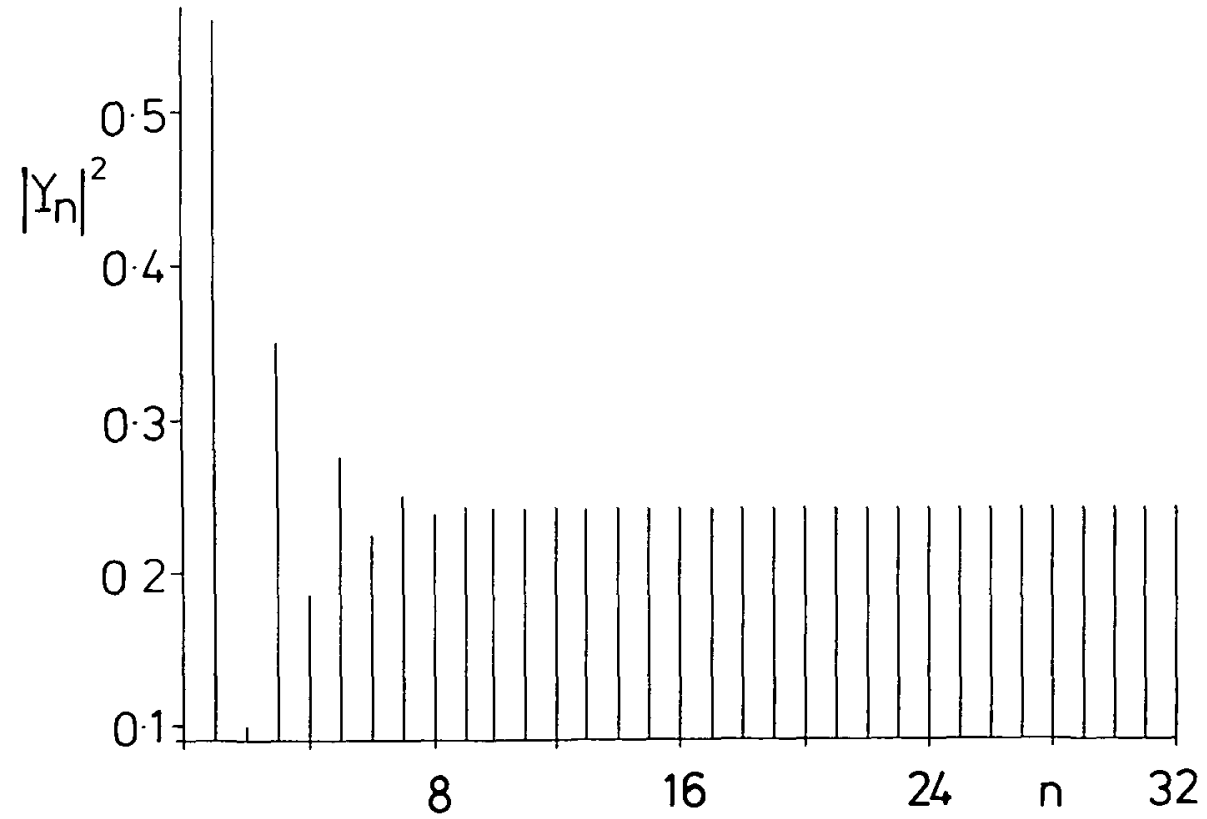

Figure 4. Light intensity $\left|Y_{n}\right|^{2}$ as a function of $n$. The fibre transmission $e^{-\alpha L}=0.7$, the coupler is specified by $C z=2$, and the linear phase shift is set at $\xi_{L}=2$.

(a) $E_{\text {pump }}=1.8$-equilibrium is reached;

(b) $E_{\text {pump }}=2.2$-the system settles to a period two pattern;

(c) $E_{\text {pump }}=4.1$-successive pulses reveal chaos. 

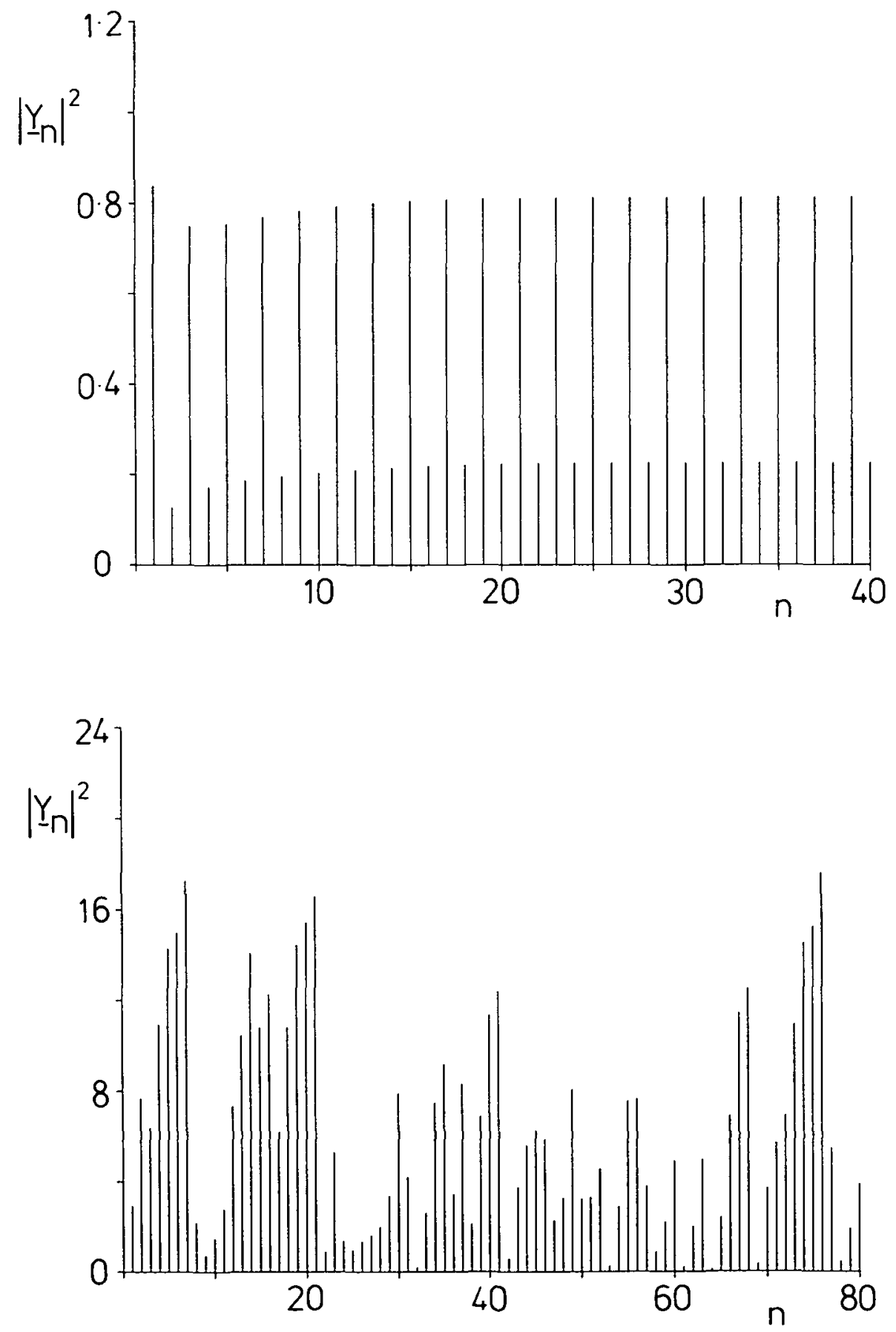

Figure 4 (continued). 

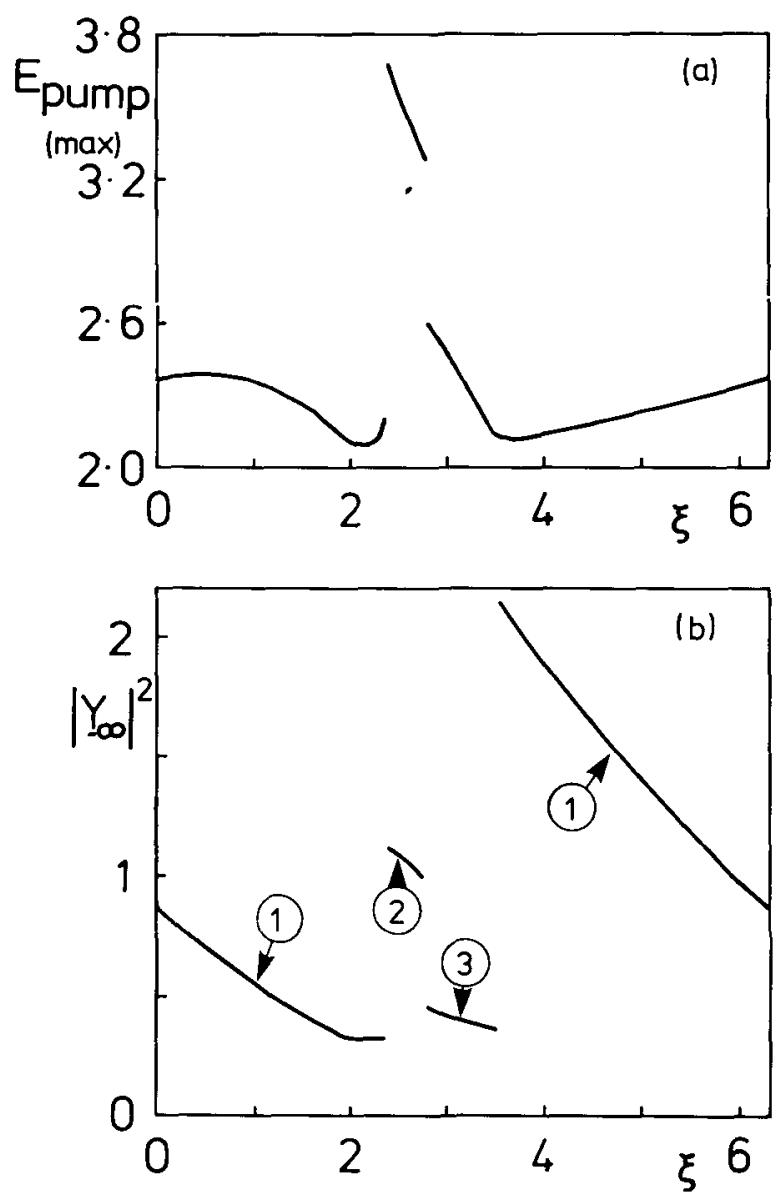

Figure 5. (a) Maximum value of $E_{\text {pump }}$ for which equilibrium is reached (after a transient), as a function of the linear phase change $\left(0 \leqslant \xi_{L} \leqslant 2 \pi\right)$. We have set $C z=2$ and $e^{-\alpha L}=0.7$ as before.

(b) Values of equilibrium output pulse intensity $\left|Y_{\infty}\right|^{2}$ at the maximum value of $E_{\text {pump }}$ (given in (a)). This again is a function of $\xi_{L}$. The function is discontinuous because the reason for the loss of equilibrium differs in each of regions 1,2 and 3, as explained in the text. The curves for region 3 cannot be predicted easily using the stability theory, whereas this is possible in regions 1 and 2 . The region 3 results depend on the starting point (in the case the origin) and do not involve loss of stability of a fixed point.

In order to characterise the onset of nonlinear behaviour, we calculate the input pump value at which the output changes from linear or fixed point behaviour for a range of fibre loop values: mathematically, we fix $B$ and $q$ and then increase $E_{\text {pump }}$ until, at $\left(E_{\text {pump }}\right)_{\max }$, a change in output occurs. The results are shown in Figure 5(a) for all possible values of the loop phase parameters $\xi_{L}, 0 \leqslant \xi_{L} \leqslant 2 \pi$. In Figure 5(b) we show the values of $\left|Y_{\infty}\right|^{2}$ obtained with $A$ just less than $A_{\max }$. There are three regions of $\xi_{L}$ values, indicated on the figures, which lead to different results. 
Region 1. The stability eigenvalues $\lambda$, (37), are real and one of them has magnitude unity at $\left(E_{\text {pump }}\right)_{\max }$. Below $\left(E_{\text {pump }}\right)_{\max }$ the iterates converge to a stable fixed point $(|\lambda|<1)$, while above it the fixed point is unstable $(|\lambda|>1)$ and period 2 behaviour occurs.

Region 2. The stability eigenvalues $\lambda$ are complex below $\left(E_{\text {pump }}\right)_{\max }$ but on reaching it two fixed points coalesce and then disappear, as discussed in the previous section and in Figure 3(a). Above $\left(E_{\text {pump }}\right)_{\max }$, the iterates of our map fall on a strange attractor.

Region 3. The stability eigenvalues are complex so that below $\left(E_{\text {pump }}\right)_{\max }$ the iterates converge to a stable point. Above $\left(E_{\text {pump }}\right)_{\max }$ our particular initial conditions, (14), do not fall within the basin of attraction of this stable fixed point and again the iterates of (12) fall on a strange attractor.

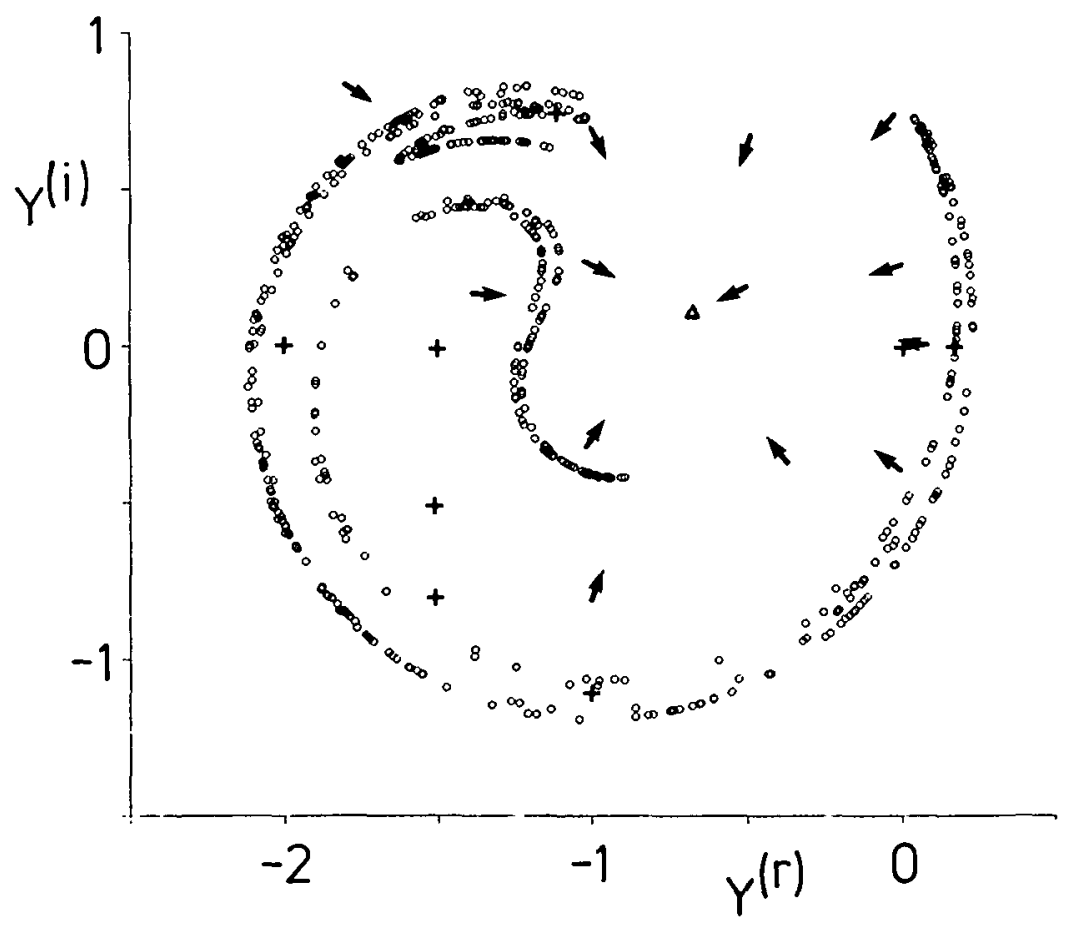

Figure 6. (a) Example of strange attractor (in the $Y$ plane). We use coupler parameters of Figure 5 , set $\xi_{L}=2.8, E_{\text {pump }}=2.64$, and omit the transient. Starting at the origin $(0,0)$ or at any of the points marked with a cross leads to successive iterates moving about forever on the attractor. By considering distances from the origin, it is evident that $\left|Y_{n}\right|^{2}$ has a minimum of about 0.03 and a maximum of around 4.5. Initial conditions marked with a small arrow lead to the fixed point $Y_{f p}=(-.6758,0.1025)$, marked with a triangle, and would produce equilibrium.

(b) Pulse intensities for the parameters given in (a). We have a chaotic sequence with intensities between the limits mentioned above. 


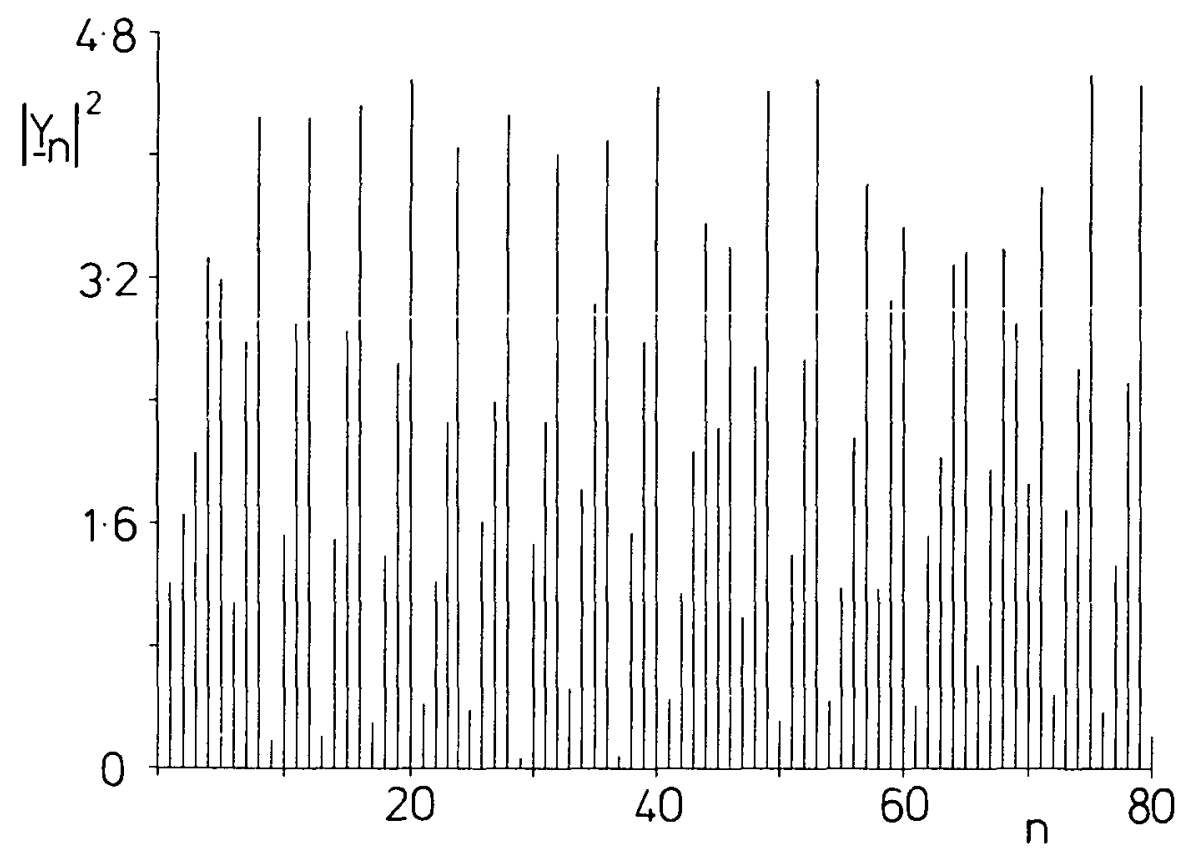

Figure 6 (continued).

The Region 3 behaviour is further illustrated in Figure 6 which shows the strange attractor developed using the initial conditions (14). The location of a stable fixed point is also indicated and some of the initial conditions giving iterates which converge to that point are also shown. Figure 6(b) shows the corresponding sequence of pulse intensities obtained from initial condition $(0,0)$. Although the map (12) may be inverted (see Appendix B) it is not simple to define the basin of attraction for a given fixed point - we refer the reader to [9] which is concerned with more technical aspects of a map of the type considered here.

Finally, in Figure 7 we show an example of results which go beyond the onset of nonlinear behaviour. If $A$ is continually increased, while other parameters are held fixed, a series of bifurcations is observed leading to period doubling behaviour. By choosing different loop and coupler parameters a great variety of diagrams of this type may be obtained involving bifurcations and discontinuous jumps onto new solutions. 


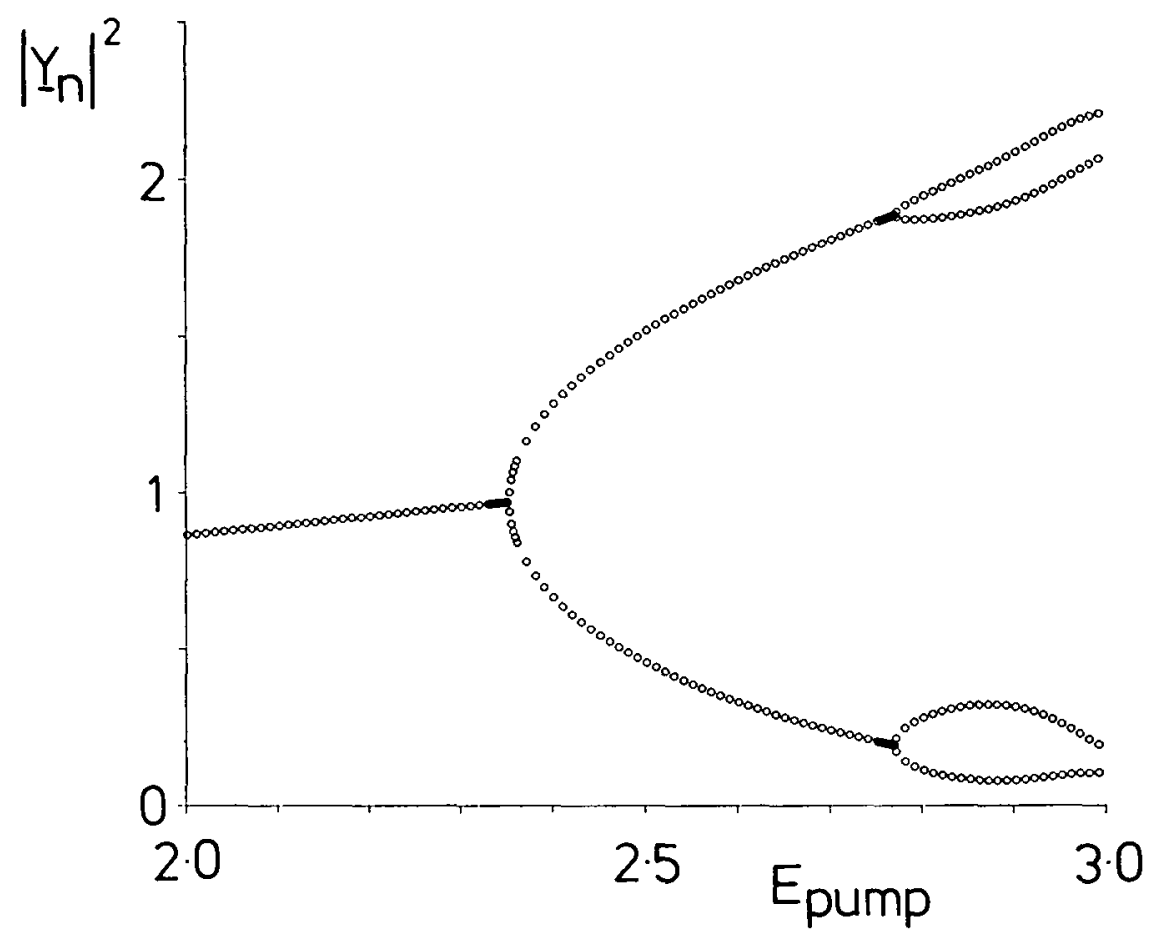

Figure 7. Illustration of bifurcations occurring as the pump field, $E_{\text {pump }}$ is increased. Here we have chosen $C z=2, e^{-\alpha L}=0.7$ and $\xi_{L}=6.0$.

\section{Conclusion}

The map which we have studied here refers to the fibre loop system as shown in Figure 1 with an infinite coherence length assumed for the laser field $E_{\text {pump. }}$. This represents one limiting situation and complements the incoherent case presented in [16]. In practice, it will be necessary to examine particular lasers and loop lengths to decide which limit, if either, is appropriate.

We have been particularly concerned with the onset of nonlinear behaviour and the results in Sections 5 and 6 demonstrate the control exerted by the system parameters. The output from the system being considered may be quite regular or may exhibit period doubling and become chaotic, with the loop phase parameter $\xi_{L}$ playing a crucial part in deciding what changes occur with increasing pump power, as illustrated in Figure 5.

The classic two-dimensional mapping paper of Hénon [10] uses a quadratic term to introduce the nonlinearity. If we assume his parameter $b$ to be positive and make the scale transformation $y \rightarrow \sqrt{ }$ 'by, the map becomes

$$
\left(\begin{array}{l}
x_{i+1} \\
y_{i+1}
\end{array}\right)=\left(\begin{array}{c}
1-a x_{i}^{2} \\
0
\end{array}\right)+\sqrt{ } b\left(\begin{array}{ll}
0 & 1 \\
1 & 0
\end{array}\right)\left(\begin{array}{l}
x_{i} \\
y_{i}
\end{array}\right) .
$$


In this case the new vector is obtained from the old one by reflection through the $y=x$ line, scaling by $\sqrt{ } b$ and then adding the vector $\left(1-a x_{1}^{2}, 0\right)$. This contrasts with our case, (12), which involves a rotation rather than a reflection, and which has the nonlinearity controlling the rotation rather than the final shift.

The map (12) could be used to define iteration procedures according to how the nonlinearity is introduced through the dependence of $\underline{A}, \underline{B}$ and $\theta_{n}$ on $\underline{Y}_{n}$. In that sense, we have begun with one of the simplest cases, buit one which has a clear physical interpretation.

The optical strange attractors which have arisen, beyond equilibrium, in the analysis of this paper, are examples of order arising where it is not expected-an order which has details not predictable in advance.

\section{Appendix A}

A light pulse of high intensity $I$ in a fibre causes the refractive index to increase by $\Delta n=\gamma I$, where $\gamma \approx 3 \times 10^{-16} \mathrm{~cm}^{2} / W$ for glasses [13] and $I$ is in $W / \mathrm{cm}^{2}$. For constant intensity light of wavelength $\lambda$, the phase change thus induced in a length $L$ is

$$
\Delta \phi=\frac{2 \pi}{\lambda} L \gamma I .
$$

In the fibre loop, $I$ is not constant, and so we find, through integration, that

$$
\Delta \phi=\frac{2 \pi}{\lambda} \gamma I_{\max } L_{f},
$$

where $I_{\max }$ is the maximum power $P$ divided by the effective area, $A_{f}$, and $L_{f}$ is the effective length. This means

$$
\frac{\Delta \phi}{P}=\frac{2 \pi}{\lambda} \gamma \frac{L_{f}}{A_{f}} \equiv 2 q .
$$

For the parameters of [8] this gives $q=1.5625 \mathrm{~W}^{-1}$, the value we use whenever the numerical value is needed in computations. If the maximum power in the loop is one watt, then the phase change is about $\pi$ radians, indicating that typical powers can lead to significant non-linear effects. We can now represent this phase change in terms of the field at the output of fibre 1 :

$$
\Delta \phi=q\left|\underline{E}_{1, n}^{(0)}\right|^{2} .
$$

This is the non-linear part of the phase change (see (5) and (10)). 


\section{Appendix B}

We prove two general results. The map (12) can be written as

$$
\underline{Y}_{n+1}-\underline{A}=B \underline{\underline{R}}\left(\theta_{n}\right)\left(\underline{Y}_{n}-\underline{A}\right)+B \underline{\underline{R}}\left(\theta_{n}\right) \underline{A} \text {. }
$$

Thus

$$
\left|\underline{Y}_{n+1}-\underline{A}\right| \leqslant|B|\left|\underline{Y}_{n}-\underline{A}\right|+|B A|
$$

This means that

$$
\left|\underline{Y}_{n+1}-\underline{A}\right| \leqslant\left|\underline{Y}_{n}-\underline{A}\right|
$$

whenever

$$
|B| \cdot\left|\underline{Y}_{n}-\underline{A}\right|+|B A| \leqslant\left|\underline{Y}_{n}-\underline{A}\right|
$$

i.e., when

$$
\left|\underline{Y}_{n}-\underline{A}\right| \geqslant|B A| /(1-|B|) \equiv \rho .
$$

Therefore, whenever $\left|\underline{Y}_{n}-\underline{A}\right|$ obeys (B5), we know from (B3) that $\underline{Y}_{n+1}$ is not further from $\underline{A}$ than is $\underline{Y}_{n}$. Then, when

$$
\left|\underline{Y}_{n}-\underline{A}\right| \leqslant \rho,
$$

(B2) implies that also

$$
\left|\underline{Y}_{n+1}-\underline{A}\right| \leqslant \rho .
$$

These results prove the invariant disc result stated in Section 3.3.

The basic map (12) can also be written as

$$
\underline{Y}_{n}=B^{-1} \underline{\underline{R}}\left(-\theta_{n}\right)\left(\underline{Y}_{n+1}-\underline{A}\right)
$$

and since

$$
\left|\underline{Y}_{n+1}-\underline{A}\right|^{2}=B^{2}\left|\underline{Y}_{n}\right|^{2}
$$

we may write

$$
\phi_{n+1} \equiv-\theta_{n}=-\xi_{L}-q B^{-2}\left|\underline{Y}_{n+1}-\underline{A}\right|^{2} .
$$

Thus we obtain the inverted map

$$
\underline{Y}_{n}=B^{-1} \underline{\underline{R}}\left(\phi_{n+1}\right)\left(\underline{Y}_{n+1}-\underline{A}\right)
$$

\section{References}

[1] A. Ankiewicz and C. Pask, "Regular and irregular motion: New mechanical results and fibre optics analogies", J. Phys. A 16 (1983) 3657-3673.

[2] A. Ankiewicz and C. Pask, Optics Letters (to be submitted).

[3] C. M. Bender and S. A. Orszag, Advanced Mathematical Methods for Scientists and Engineers (McGraw-Hill, 1978).

[4] J. Bernussou, Point Mapping Stability (Pergamon Press, 1977). 
[5] M. V. Berry, "Regular and irregular motion", in Topics in nonlinear dynamıcs, AIP Conf. Proc. 46 (AIP, New York, 1978) 16 -120.

[6] R. J. Black and A. Ankiewicz, "Fibre-optic analogies with mechanics", Amer. J. Phys. 53 (1985) 554-563.

[7] C. M. Bowden, H. M. Gibbs and S. L. McCall (eds.) Optical Bistability 2 (Plenum Press, New York, 1982).

[8] E. Desurvire, M. Digonnet and H. J. Shaw, "Raman amplification of recirculating pulses in a reentrant fiber loop", Optics Letters 10 (1985) 83-85.

[9̈j 5. M. Hiammei, C. K. R. T. Jones and J. V. Moloney, "Global dynamical behaviour of optical field in a ring cavity", J. Opt. Soc. Amer. B 2 (1985) 552-564.

[10] M. Hénon, "A two-dimensional mapping with a strange attractor", Comm. Math. Phys. 50 (1976) 69-77.

[11] K. P. Jackson et. al., "Optical fiber delay-line signal processing", IEEE Trans. Microwave Theory Tech. 33 (1985) 193-210.

[12] P. D. Maker and R. Terhune, "Study of optical effects due to an induced polarization third order in the electric field strength", Phys. Rev. 137 (1965) A801-818.

[13] D. Milam and M. J. Weber, "Measurement of nonlinear refractive-index coefficients using time resolved interferometry: Application to optical materials for high-power neodymium lasers", $J$. Appl. Phys. 47 (1976) 2497-2501.

[14] A. W. Snyder and J. D. Love, Optical Wavegude Theory (Chapman and Hall, 1983).

[15] L. F. Stokes, M. Chodorow and H. J. Shaw, "All-single-mode fibre resonator", Optics Letters 7 (1982) 288-290.

[16] M. Tur, B. Moslehi and J. W. Goodman, "Theory of laser phase noise in recirculating fibre-optic delay lines", IEEE J. Lightwave Tech. 3 (1985) 20-31. 